Editorial

\title{
Representación y Gestión del Conocimiento
}

\section{Representation and Knowledge Management}

\author{
Adolfo Lozano-Tello ${ }^{1}$, Álvaro Rocha ${ }^{2}$ \\ alozano@unex.es, amrocha@dei.uc.pt \\ ${ }^{1}$ Universidad de Extremadura . Grupo Quercus SEG, Escuela Politécnica de Cáceres. Avda de la Universidad \\ sn, 10003 Cáceres, Spain. \\ ${ }_{2}^{2}$ Universidade de Coimbra, Departamento de Engenharia Informática, Pólo II - Pinhal de Marrocos, 3030- \\ 290 Coimbra, Portugal.
}

DOI: 10.17013/risti.14.vii-viii

\section{Introdução}

La gestión del conocimiento en sistemas de información toma cada día más importancia sobre todo en sistemas y problemas complejos en los que es fundamental organizar y analizar la información con técnicas adecuadas. En algunos campos, como los procesos de desarrollo del software o en el campo de la salud es fundamental establecer metodologías idóneas que hagan más eficientes estos sistemas.

En este número 14 de la Revista Ibérica de Sistemas y Tecnologías de la Información (RISTI) dedicado a la "Representación y Gestión del Conocimiento", nos ha sido muy grato recopilar artículos relevantes que aportan soluciones a procesos de gestión del conocimiento aplicado a diferentes áreas, $\mathrm{y}$ aportaciones significativas de representación del conocimiento integradas a los procesos de gestión.

Los 7 artículos que componen este número se han seleccionado de un total de 55 artículos enviados, por lo que el índice de selección ha sido de un 12.7 \%, un ratio muy significativo que, lamentablemente, deja fuera algunas aportaciones muy interesantes que también podrían haber formado parte de este número temático.

\section{Estructura}

En los artículos seleccionados, en el primer bloque se proponen soluciones de la gestión del conocimiento en el campo de la salud. En el segundo bloque se tratan aspectos sobre la gestión de procesos de desarrollo de software y workflows, y en uso de ontologías en estos sistemas. 
El primero de los artículos, "Arquitetura de Informação de Suporte à Gestão da Qualidade em Unidades Hospitalares”, propone una completa solución mediante una arquitectura de información, sobre la gestión de calidad de las organizaciones centradas en la asistencia sanitaria.

En el siguiente artículo,"TICE.Healthy: Integração de soluções TIC para a 'Saúde e Qualidade de Vida'”, también en el campo de la salud, se propone la plataforma web Evida para la gestión del conocimiento y la integración de información de diferentes proyectos.

El tercer artículo, "O impacto da exclusão digital na utilização potencial de um mercado eletrónico de serviços de cuidados de saúde e serviços sociais", se presenta un estudio y análisis del impacto de las TICs en servicios sociales y de salud.

El cuarto, "Análisis de Flujos de Conocimiento en Proyectos de Mejora de Procesos Software bajo una perspectiva multi-enfoque", ya en el bloque sobre la gestión de desarrollos de software, propone una estrategia para la gestión del conocimiento en la mejora de procesos software.

El quinto artículo, "Adaptación de Workflows basada en Ontologías", proporciona un completo método de representación y propagación de cambios en workflows de procesos administrativos representados mediante ontologías.

El sexto artículo, "Evaluación empírica del mapeo de reglas de negocio para el desarrollo de ontologías" aporta reglas de mapeo desde SBVR (utilizado en la Ingeniería del Software) a OWL2 (utilizado en la Ingeniería Ontológica).

Por último, el artículo "Representación del Conocimiento de la Información Geográfica siguiendo un Enfoque basado en Ontologías", presenta una propuesta de aplicación práctica de uso de ontologías a los sistemas de información geográfica.

\section{Agradecimientos}

Queremos agradecer a la AISTI (Asociación Ibérica de Sistemas e Tecnologías de Información) la oportunidad de difundir los trabajos más relevantes que se han enviado a este número temático, y sobre todo al Comité Científico por haber hecho el esfuerzo de revisar la importante cantidad de contribuciones. Asimismo, a los autores de los artículos por haber enviado sus trabajos a la revista. Con este grado de participación, estamos seguros de que tendremos la oportunidad de hacer otro número con una temática similar. 